\title{
ADDITIVE LOCAL MULTIPLICATIONS AND ZERO-PRESERVING MAPS ON $C(X)$
}

\author{
QIAN HU
}

Abstract. Suppose $X$ is a compact Hausdorff space. In terms of topological properties of $X$, we find topological conditions on $X$ that are equivalent to each of the following: 1 . Every additive local multiplication on $C(X)$ is a multiplication, 2. Every additive local multiplication on $C_{R}(X)$ is a multiplication, 3. Every additive map on $C(X)$ that is zero-preserving (i.e., $f(x)=0$ implies $(T f)(x)=0)$ has the form $T(f)=T(1) \operatorname{Re} f+T(i) \operatorname{Im} f$.

Mathematics subject classification (2010): Primary 47B48, 54C45; Secondary 54D30, 16 S99. Keywords and phrases: Local multiplications, zero-preserving maps, F-spaces, $\mathfrak{q}$-points, P-points.

\section{REFERENCES}

[1] E. Christensen, Derivations of nest algebras, Math. Ann. 229 (1977) 155-161.

[2] R. CRIST, Local derivations on operator algebras, J. Funct. Anal. 135 (1996) 76-92.

[3] L. Gillman, M. Henriksen, Concerning Rings of Continuous Functions, Trans. Amer. Math. Soc. (1954) 340-362.

[4] L. Gillman, M. Jerison, Rings of continuous functions, Graduate Texts in Mathematics, No. 43, Springer-Verlag, New York-Heidelberg, 1976.

[5] Hadwin, Don Algebraically reflexive linear transformations, Linear and Multilinear Algebra 14 (1983) 225-233.

[6] The Hadwin Lunch Bunch, Local multiplications on algebras spanned by idempotents, Linear and Multilinear Algebra 37 (1994) 259-263.

[7] D. HADWIn, J. W. KeRR, Local multiplications on algebras, J. Pure Appl. Algebra 115 (1997) 231239.

[8] D. Hadwin, J. Li, Local derivations and local automorphisms, J. Math. Anal. Appl. 290 (2004) 702-714.

[9] D. HADWIN, J. LI, Local derivations and local automorphisms on some algebras, J. Operator Theory 60 (2008) 29-44.

[10] D. Han, S.-Y. WeI, Local derivations of nest algebras, Proc. Amer. Math. Soc. 123 (1995) 30953100.

[11] R. V. KADison, Local derivations, J. Algebra 130 (1990) 494-509.

[12] A. K. MisRA, A topological view of P-spaces, General Topology and its Applications 2 (1972) 349_ 362.

[13] W. Rudin, Homogeneity problems in the theory of Čech compactifications, Duke Math. J. 23 (1956) 409-419.

[14] P. ŠEMRL, Local automorphisms and derivations on B(H), Proc. Amer. Math. Soc. 125 (1997) $2677-$ 2680.

[15] S. Willard, General topology, Addison-Wesley Publishing Co., Reading, Mass.-London-Don Mills, Ont. 1970.

[16] E. Wimmers, The Shelah P-point independence theorem, Israel J. Math. 43 (1982) 28-48. 